\title{
El agua en el jardín inglés dieciochesco
}

\author{
Ibeas-Altamira, Juan Manuel
}

Universidad del País Vasco (UPV-EHU), juan.ibeas@ehu.es

\begin{abstract}
Resumen
Pintoresco, pastoral, agradable, metafórico, delicioso - he aquí algunos de los adjetivos que describen el jardín paisajístico inglés, que triunfó en Europa en el siglo XVIII superando el modelo del jardín a la francesa. Era la expresión de una época, que seguía al barroco y que soñaba con el romanticismo y con la libertad. Dichos espacios presentan una naturaleza ideal inspirada en las pinturas de Nicolas Poussin y Claude de Lorraine así como en los jardínes chinos clásicos descritos por los viajeros. En todos ellos el agua es el elemento principal, no solo en su estado líquido: arroyos, estanques, torrentes, cascadas, lagos, sino también gaseoso creando toda una atmósfera.
\end{abstract}

Palabras clave: agua; jardín inglés; literatura; siglo XVIII.

\section{Résumé}

Pittoresque, pastoral, agréable, métaphorique, délicieux- voilà quelques adjectifs qu'on emploie pour décrire le jardin paysager anglais, qui triompha en Europe au XVIIIème siècle laissant de côté le 'jardin à la française'. C'était l'expression d'une époque, qui suivait le baroque et qui rêvait de romantisme et de liberté. Ces espaces présentent une nature idéale inspirée par les peintures de Nicolas Poussin et Claude de Lorraine ainsi que par les jardins chinois classiques décrits par les voyageurs. Dans tous ces espaces l'eau est l'élément principal, non seulement à l'état liquide: ruisseaux, bassins, torrents, chutes, lacs; mais aussi gazeux créant une atmosphère.

Mots-clés: eau; jardin anglais; littérature; XVIIlème siècle.

\begin{abstract}
Picturesque, pastoral, pleasant, metaphorical, delightful -these are some of the adjectives that describe the eighteenth-century English landscape garden that spread across Europe, replacing the symmetrical jardin à la française. It was an expression of a period in time, following baroque and looking ahead to romanticism in its freedom from strict rules. Inspirated by de drawings of Nicolas Poussin and Claude Lorraine and by the Chinese classic gardens described by travelers it presented an idealized view of nature. In all of them water is the most important element, but not only liquid: streams, lagoons, watercourses, falls, lakes; also as gas creating an atmosphere.
\end{abstract}

Keywords: Water; english garden; XVIII th century. 
El jardín del siglo XVIII se halla marcado por un regreso al paisaje arcádico de los antiguos griegos, al paisaje de la roma clásica y a la mitología que aparecía retratado en las pinturas de Claude Lorrain o Poussin. Iluminada por la luz de la filosofía y la pintura toda Europa se entusiasma con los jardines: los filósofos meditan en ellos, los poetas los cantan y los aristócratas los cultivan. Es en Inglaterra donde nace el nuevo modelo de jardín que redescubre la naturaleza a través de la pintura: es lo que se va a denominar el jardín paisajista, o jardín inglés, o chino o incluso anglo-chino. Si toda Europa está de acuerdo en el deseo de cambio, la cronología demuestra que el nuevo estilo irregular apareció al otro lado de La Mancha. Ya en 1734 podemos leer una carta de sir Thomas Robinson que afirma que toda la isla se halla entusiasmada imitando los caprichosos jardines del príncipe de Gales (Brownell, 1978: 175).

Además de a la irregularidad, en este nuevo espacio se da una particular importancia a los puntos de vista teatrales (no sorprende por lo tanto que muchos de sus iniciadores tuvieran vinculaciones con el teatro), a perspectivas sobrecogedoras sobre una naturaleza desbordante, sin cercar y, en apariencia, no domesticada; y así se hará destacar los elementos particulares del lugar original , como un árbol curioso, un césped deslumbrante, una gruta o unas rocas, y el modo en que todo ello se ha de subrayar es recurriendo a un elemento clave de dicho universo: el agua, que como no podía ser de otro modo va a ser tratada de un modo muy particular, para conseguir unos novedosos efectos. Por ello Alexander Pope (16881744) en su epístola a Burlington aconsejaba dejarse guiar por el genio del lugar, es decir poner de relieve la esencia propia del lugar, ya que ese «genio» había de dibujar mientras plantábamos:

Consult the Genius of the Place in all,

That tells the Waters or to rise, or fall,

Or helps th' ambitious Hill the Heav'ns to scale,

Or scoops in circling Theatres the Vale,

Calls in the Country, catches opening Glades,

Joins willing Woods, and varies Shades from Shades,

Now breaks, or now directs, th' intending Lines;

Paints as you plant, and as you work, Designs (Pope, 1731: 7).

En el origen se trató de un movimiento esencialmente literario y así, las primeras condenas a la artificialidad de los jardines franceses las encontramos en los textos de John Milton (1608-1674), del conde de Shaftesbury (1671-1713), de Joseph Addison (1672-1719) y del poeta Alexander Pope (1688-1744). Pensemos en ese célebre fragmento del Paraíso perdido de Milton, de 1667, que nos presenta un jardín del Edén en nada regular y abundantemente regado por un inmenso rio; no puede ser visto más que como una apertura al nuevo jardín del XVIII:

Por el lado del Mediodía, y a través del Edén, pasaba un anchuroso río, que no variaba su curso, sino que se sepultaba bajo la escarpada montaña. Dios había colocado aquella montaña, como el suelo de su elevado jardín, sobre la rápida corriente. La onda atraída hacia lo alto por la dulce sed de la tierra porosa, brotaba de sus venas como límpida fuente y se desparramaba por el jardín (Milton, 1667: 98)ํ․

La literatura, y en particular la poesía recurren a la descripción del paisaje como una de las simbologías más poderosas para expresar las subjetivas experiencias de la condición humana ${ }^{2}$; y podemos decir que la literatura inglesa en particular, se ha focalizado en la naturaleza para conformar una producción volcada en el medio ambiente que rodea al ser humano. Una poética curiosa e interesada en el entorno y que dio la mano a los nuevos cambios políticos dictados por la Cámara de los Comunes ('Inclosure Acts’) que permitía vallar terrenos comunales como compensación a la pérdida de derechos

\footnotetext{
${ }^{1}$ «Southward through Eden went a river large,/ Nor changed his course, but through the shaggy hill/ Passed underneath ingulfed; for God had thrown/ That mountain as his garden-mould high raised/ Upon the rapid current, which, through veins/ Of porous earth with kindly thirst up-drawn,/ Rose a fresh fountain, and with many a rill/ Watered the garden».

${ }^{2}$ Desde la tradición grecolatina encontramos obras donde el locus conforma la esencia de los personajes y el Beatus ille siempre ha sido recurrente en poesía.
} 
comunales de los pequeños propietarios ${ }^{3}$ : extensas superficies se destinaron a la creación de parques. Paradójicamente se trataba de los primeros pasos de la Revolución Industrial cantados por poemas eco-pastoriles. Las mejoras en técnicas en el transporte propias de la época animaron a los propietarios a acondicionar sus residencias campestres favoreciendo el movimiento. Además la nueva concepción de la propiedad privada dictada por John Locke, según la cual el hombre es propietario de lo que adquiere por su propio trabajo y el de sus criados, y que fue llevada por Joseph Addison al terreno de la jardinería paisajística, refuerza estas ideas, pues hasta las zonas pantanosas inundadas por el agua habían de ser provechosas:

Pero ¿por qué no convertir una propiedad en una especie de jardín con plantaciones frecuentes que acrecentaran los beneficios y el placer de su propietario? Una marisma en la que se planten sauces o una montaña bañada por la sombra de los robles no solo son más bellos sino que también resultan más rentables que si se dejan desnudos y abandonados (Addison, 1712) ${ }^{4}$.

Los nuevos parques no debían ya presentar un entorno sometido a la gloria de un hombre, sino que habían de ser los espacios de la nueva libertad y cantar subrepticiamente a los tiempos modernos, a la floreciente economía agraria que debía ser representada simbólicamente en los diferentes elementos que lo configuran: praderas que evocarán la ganadería, bosquecillos que recuerdan la madera para calentarse, el arroyo que sugerirá el espacio de pesca y el lago que será el mar que rodea a Inglaterra y la enriquece por el comercio.

Incluso las ruinas que encontramos a menudo sobresaliendo encima de lagos y rocas eran un emblema pintoresco de los valores políticos del nuevo régimen las góticas evocaban la herencia de los saxones y las libertades nórdicas y las romanas la pureza de la época clásica. Y todo este conjunto recorrido por el agua, como idea de ciclo vital, con una vida resplandeciente y una idea de muerte placida y poética: un ecosistema en aparente armonía en el que el hombre forma parte del todo. Como vasos sanguíneos los caminos y arroyos serpenteantes, recorren el tejido vivo de un jardín que siente y palpita como su público. Un espacio perfecto para la reflexión filosófica del siglo durante un paseo errático por un recorrido aleatorio.

Pero todo ello, como digo, sin caer en lo evidente pues por encima de todo reinara el espacio natural a gran escala donde se privilegia el aspecto salvaje y poético de una naturaleza que no se intenta controlar sino disfrutar. Hasta entonces la línea recta había encarnado un despotismo de rigidez extrema, y en oposición, la línea zigzagueante, la línea serpentina cantada por Hogarth en su Análisis de la Belleza, ha de ser la de las libertades. Pese a la apariencia caricaturesca por la simpleza de la idea tuvo mucho eco. Así lo vemos en Addison que en The Tatler al narrar un sueño durante el que visita Suiza (tierra de libertad donde no hay monarquía) descubre un río, el Ródano, que transcurre lento por sus numerosos meandros, regando los campos, hasta detenerse en un amplio lago; entonces, al llegar a las regiones en que reina la servidumbre, elige el camino más corto, las atraviesa con una rapidez increíble, y desemboca lo antes posible en el mar ${ }^{5}$.

Romper con formas geométricas de historia reciente para cultivar la irregularidad y encontrar el paisaje real con sus características propias modeladas por el tiempo, las lluvias y los ríos. Los teóricos del nuevo estilo no se hicieron esperar y, en 1715, Stephen Switzer (1682-1745) publicó su manual Gardener’s Recreation (que sería reeditado años más tarde como Iconographia Rustica) dando respuesta la demanda social. Poco después le seguía Batty Langley (1696-1751) con A Sure Method of Improving Estates (1728) y New Principles of Gardening (1728) un canto a lo salvaje, a lo tortuoso y al recurso al medio acuático para poetizar el conjunto.

Los primeros grandes paisajistas ingleses: Charles Bridgeman (1690-1738), William Kent (1685-1748), Lancelot Brown (1716-1783) o sir John Vanbrugh (1664-1726) se pusieron pronto manos a la obra y las primeras creaciones importantes

\footnotetext{
${ }^{3}$ Si bien el movimiento había nacido en el siglo XV, es a partir del XVIII cuando se intensifica. Este cambio explica el paso a una agricultura intensiva y de tipo capitalista al forzar a los agricultores a emplear nuevas técnicas y cultivos y al fomentar la propiedad privada. Muchos campesinos se vieron forzados a abandonar el campo y a instalarse en la ciudad (Verley, 1985: 204).

${ }^{4}$ «But why may not a whole estate be thrown into a kind of garden by frequent plantations, that may turn as much to the profit as the pleasure of the owner? A marsh overgrown with willows, or a mountain shaded with oaks, are not only more beautiful, but more beneficial, than when they lie bare and unadorned.»

${ }^{5}$ «This river after having made progress through those free nations stagnates in a huge lake at the leaving of them and no sooner enters into the regions of slavery but runs through them with an incredible rapidity and takes its shortest way the sea» (Addison, 1710).
} 
surgieron en las dos primeras décadas del siglo con los jardines de Castle Howard (1725), Claremont (1715-1727), Chistwick (1720-1727) y Blenheim (1764). La nueva corriente pronto se unió a la ola neopaladiana que, daba una importancia fundamental al agua, ya que su inspirador, el manierista Palladio ${ }^{6}$, consideraba que era un elemento que reforzaba las simetrías y delimitaba las líneas, y a menudo estructuraba sus Villas en función a la misma.

A mediados del XVIII el redescubrimiento de la China, de la mano de las cartas de los padres jesuitas que se hallaban allí como misioneros supuso el impulso definitivo a esta pasión en toda Europa. El emperador Yuanming Yuan, en su palacio de Pekín gozaba del «jardín de los jardines», un espacio creado ante todo para el placer pero que, como nos recuerda en su carta el padre Jean-Denis Attiret (1702-1768) que lo visitó, también fomentaba el comercio en los alrededores. En este nuevo Paraíso terrenal el agua fluía por doquier y regaba cada rincón:

Pour les maisons de plaisance, elles sont charmantes. Elles consistent dans un vaste terrain, où l'on a élevé à la main de petites montagnes, hautes depuis vingt jusqu'à cinquante et soixante pieds, ce qui forme une infinité de petits vallons. Des canaux d'une eau claire arrosent le fond de ces vallons, et vont se joindre en plusieurs endroits pour former des étangs et des mers. On parcourt ces canaux, ces mers et ces étangs sur de belles et magnifiques barques [...]. Dans chacun de ces vallons, sur le bord des eaux, sont des bâtiments parfaitement assortis de plusieurs corps de logis, de cours de galeries ouvertes et fermées, de jardins, de parterres, de cascades, etc., ce qui fait un assemblage dont le coup d'œil est admirable. On sort d'un vallon, non par de belles allées, droites comme en Europe, mais par des zigzags, par des circuits, qui sont eux-mêmes ornés de petits pavillons, de petites grottes, et au sortir desquels on retrouve un second vallon tout différent du premier, soit pour la forme du terrain, soit pour la structure des bâtiments.

Toutes les montagnes et collines sont couvertes d'arbres, surtout d'arbres à fleurs, qui sont ici très communs. C'est un vrai paradis terrestre. Les canaux ne sont point comme chez nous bordés de pierres de taille tirées au cordeau, mais tout rustiquement avec des morceaux de roche, dont les uns avancent, les autres reculent, et qui sont posés avec tant d'art, qu'on dirait que c'est l'ouvrage de la nature. Tantôt le canal est large, tantôt étroit : ici il serpente, là il fait des coudes, comme si réellement il était poussé par les collines et les rochers. Les bords sont semés de fleurs qui sortent des rocailles, et qui paraissent y être l'ouvrage de la nature ; chaque saison a les siennes. Outre les canaux, il y a partout des chemins, ou plutôt des sentiers, qui sont pavés de petits cailloux, et qui conduisent d’un vallon à l'autre. Ces sentiers vont aussi en serpentant ; tantôt ils sont sur les bords des canaux, tantôt ils s'en éloignent (Vissière, 2001: 230-231).

Un agua libre y desbocada que no solo aparece en su esencia líquida sino también gaseosa, es decir que, para trabajar la perspectiva atmosférica se busca generar efectos de profundidad a través de la bruma, que difumina lo más alejado y se entremezcla con el follaje graduando las tonalidades del verde: la humedad ambiente distorsiona la luminosidad generando una sensación de profundidad. Al igual que en las pinturas chinas que inundan el mercado europeo muestran bosques envueltos en brumas los jardines paisajísticos anhelan una niebla que dibuje sus contornos cambiando sin cesar. La técnica atmosférica, aunque se empleara ya en los frescos pompeyanos, se toma de la pintura inglesa, donde tal práctica es recurrente ${ }^{7}$. Con estos recursos se conseguirá el tan ansiado efecto «pintoresco»; como apuntaba pues como explicaba William Kent (1685-1748) el arte de los jardines será la pintura del paisaje, es decir, buscan plantar cuadros.

Aunque tras la Guerra de los Siete Años (1763) los viajeros empiezan a cruzar la Mancha en ambos sentidos, y en el Hexágono se deja a un lado definitivamente el restrictivo jardín a la francesa sublimados por Le Notre en Vaux-leVicomte, las Tullerías o Versalles donde las perspectivas debían ser extremadamente claras y las líneas lo más puras posibles y se buscan efectos nuevos. El público de los jardines franceses se había saturado de ese artificio que únicamente buscaba someter a la naturaleza, harto de estanques que continuaban el Salón de los Espejos y de fuentes ritmadas al paso del rey sol. Había quedado atrás la época de la «Nueva Rom que ansiaba imitar las hazañas de ingeniería de los romanos. Así vemos a modo de ejemplo que la máquina de Marly, ese artilugio ideado para subir el agua del Sena a las fuentes del palacio y que en la época había causado furor ${ }^{8}$, se empieza a contemplar con espanto. Hasta en poesía encontramos

\footnotetext{
${ }^{6}$ Andrea Palladio, en su obra Los cuatro libros de la arquitectura (en italiano, I quattro libri dell'architettura) de 1570 subrayaba esta idea.

${ }^{7}$ Se había descubierto que el polvo y la humedad en el ambiente causaban la dispersión de la luminosidad; siendo la luz de longitud de onda corta (azul) más diseminada y la luz de longitud de onda larga (roja) menos esparcida. El nombre de perspectiva atmosférica le viene de Leonardo da Vinci.

${ }^{8}$ Existe un poema de M. Cassan, publicado en el Mercure galant de 1699, que canta la llegada de la ninfa del Sena al palacio de Marly, y expone como esta es tranquilizada por Apolo y acogida por Luis XIV: «Ainsi se voit de loin la machine effroyable,/ Ouvrage de nos jours, qui paraît incroyable.[...]/ Quoi ! dit-elle en voyant la machine étonnante,/ Serai-je donc contrainte à poursuivre ma pente ,/ Et me faire rouer parmi tous les ressorts/ Que je vois remuer par de si grands efforts!/ Non, non, dit-elle alors,
} 
ejemplos de tal cambio cuando leemos lo que escribió Elizabeth-Francoise-Sophie de la Live, condesa de Houdetot, en 1778 en el castillo de Fourqueux:

\author{
SUR LA MACHINE DE MARLY \\ Cet appareil de fer et ces grands mouvements, \\ Ces efforts redoublés et ces gémissements \\ Offrent partout aux sens la nature offensée; \\ Elle semble gémir d'avoir été forcée, \\ Et, cédant à regret aux entraves de l'art, \\ Aux caprices des rois se plaint d'avoir en part (Buffenoir, 1901: 63).
}

A partir de la década de 1770 Francia se lanza a la nueva moda con entusiasmo y llega incluso al gran público generando un amplio debate. El influjo de Rousseau (1712-1778) Schiller y Goethe acabaría de inclinar la balanza dando el triunfo a un paisaje sublime que debía invitar a la reflexión. A ambos lados del canal surge toda una literatura con grandes nombres como Chambers, Chabanon, Le Rouge, Girardin, Watelet, More, Monville etc. El nuevo universo paisajístico inunda poco a poco Alemania, Italia, Polonia y Rusia extendiéndose poco a poco esos microecosistemas regados por arroyos que acogían un palpitante lago en su interior.

En Francia marcarán tendencia el nada desértico Desierto de Retz (de Monville), Ermenonville (de Girardin) con su islatumba de Rousseau diseñada por Hubert Robert, el parque Monceau con su Naumaquia (un estanque ovalado rodeado por columnas corintias) (de Carmontelle), la Aldea de la Reina de Versalles y la Folie de Saint-James (de Bélanger). En todos estos espacios el agua se vuelve indispensable para una bella composición hasta el punto en que tanto las grandes extensiones agrícolas como los pequeños espacios ciudadanos no pueden entenderse sin tenerla como protagonista, invitando tanto a la melancolía como sobrecogiendo los ánimos. Acomodándose a todas las situaciones se vuelve el elemento más interesante del paisaje, y como esencia vital que recorre estos jardines contribuye a la tranquilidad y a la meditación de sus visitantes. Son color y sombra, fuente de destellos y de frescor, son canto y son silencio.

\title{
Referencias bibliográficas
}

AdDison, Joseph (18-20 de abril de 1710). En The Tatler, nº 161.

AdDison, Joseph (25 de junio de 1712). «On the Pleasures of the imagination» en The Spectator, $\mathrm{n}^{\circ} 424$.

Brownell, Morris R. (1978). A. Pope and the Arts in Georgian England. Londres: Oxford University Press.

BuffENoIR, Hippolyte (1901). La Comtesse d’Houdetot. París: Calmann Lévy.

CASSAN, Marly (1699). La nymphe de Chanceaux, ou L'arrivée de la Seine au château de Marly. París: A. Chrétien.

MiLton, John (2007). Paradise Lost. Londres: Blackwell Publishing.

Pope, Alexander (1731). An epistle to the Right Honourable Richard Earl of Burlington. Occasion'd by his publishing Palladio's designs of the baths, arches, theatres, \&c. of ancient Rome. Londres: L. Gilliver.

Verley, Patrick (1985). La révolution industrielle. París: MA éditions.

VISSIERE, Isabelle y Jean-Louis (2001). Lettres édifiantes et curieuses des jésuites de Chine (1702-1776). París: Desjonquères.

la nymphe de la Seine/ Se mêlera plutôt avec l'eau qui l'entraîne,/ Et, par son changement, saura bien éviter/ Les outrages cruels qu'elle voit apprêter» (Cassan, 1699: 1011). 